\title{
Midline Mandibulotomy Approach For Oral Cavity And Oropharynx Tumors: Long-Term Experience In A Single Institution
}

José M López-Arcas

Alfonso X el Sabio University (Madrid, Spain)

Juan Manuel Vadillo ( $\sim$ jumava10@gmail.com )

Alfonso X el Sabio University (Madrid, Spain)

José L Del Castillo

University Hospital La Paz (Madrid, Spain)

Patricia A Lara

Alfonso X el Sabio University (Madrid, Spain)

José L. Cebrián

University Hospital La Paz (Madrid, Spain)

Beatriz Ruiz-Sánchez

Alfonso X el Sabio University (Madrid, Spain)

Juan M. Aragoneses

Alfonso X el Sabio University (Madrid, Spain)

\section{Research Article}

Keywords: mandible osteotomy, midline approach, oropharynx, oral cavity tumors.

Posted Date: January 24th, 2022

DOI: https://doi.org/10.21203/rs.3.rs-1159348/v2

License: (9) This work is licensed under a Creative Commons Attribution 4.0 International License.

Read Full License 


\section{Abstract}

Purpose: To describe our clinical experience with the use of the midline mandibulotomy approach for oral cavity and oropharynx tumors.

Methods: Charts were reviewed retrospectively for 67 consecutive patients who underwent mandibulotomies over a 15-year period (2002-2017) as part of their treatment for oral and oropharyngeal malignancies, with an average follow-up of 57.7 months.

Results: Sixty-seven patients underwent a mandibulotomy. There were 59 males (88\%) with a mean age of 56.9 years and eight females $(12 \%)$ with a mean age 56.5 years. The approach was a midline mandibulotomy in 50 patients (74.6\%), a paramedian mandibulotomy in 10 patients (14.9\%), and a posterior mandibulotomy in seven (10.44\%: angle $1(1.5 \%)$, body $5(7.5 \%)$, and ramus $1(1.5 \%)$.

In the group of patients undergoing median or paramedian mandibulotomies, adequate exposure of the lesion was achieved in all cases with a significant lower rate of complications $(13,4 \%)(p<0.005)$ compared to the posterior mandibulotomy group $(37,5 \%)$.

Conclusions: The results of the study confirm that the anterior mandibulotomy approach provides excellent exposure for oral and oropharyngeal tumors, with a significant lower complication rate compared to the posterior mandibulotomy approach.

Until minimally invasive Robotic technology is not widespread, conventional techniques such as midline mandibulotomy approaches, with "modern-times" refinements still have their place in head and neck oncology surgery

\section{Background}

Since its first description in 1836 by Roux [1], different variations of the midline mandibulotomy approach for tumors of the oral cavity and oropharynx have been used. In the 1980s, based on the studies published by Spiro et al. [2, 3], the midline approach was modified and lateral and paramedian approaches, with improved theoretical features, were adopted. Subsequent studies have analyzed the advantages and disadvantages of each approach [4-6].

In general, mandibular splitting is indicated for tumors of the tonsils, base of the tongue, and the retromolar fat pad, without clinical or radiological findings of bony invasion, to provide good surgical access to the primary lesion and facilitate "en-bloc" resection.

This technique can be divided grossly into anterior and lateral/posterior approaches, according to whether the osteotomy is anterior or posterior to the mental foramen. A posterior mandibulotomy has several disadvantages: one of the most important being the higher incidence of osteoradionecrosis, due to the location of the osteotomy in the radiotherapy field. In addition, the inferior alveolar nerve is often damaged, and this method gives less access than the anterior approach (4). Therefore, it has generally 
been abandoned. The median mandibulotomy can be further classified into the midline mandibulotomy between the two central incisors, and the para-midline mandibulotomy between a lateral incisor and canine.

The median mandibulotomy approach begins by designing the skin incision (Figure 1). Different designs have been used, none of them proven superior to others, however special attention must be taken in the lip-skin mucosal junction to prevent poor lip scarring.

To perform the mandibular symphysis osteotomy we use a stair-case design (Figure 2). A thin-saw blade with abundant irrigation is used to perform the osteotomy. The lingual cortex osteotomy can be completed by means of a chisel or a Piezosurgery Device (Mectron $\left.{ }^{\circledR}\right)$ to minimize the damage to the sof tissues of the floor of the mouth.

Osteosynthesis is done by two "locking" $2.0 \mathrm{~mm}$ miniplates separated one from another at least 2-3 cm (Figure 3). Reattachment of the genioglossal/geniohyoid muscles is of outmost importance.

This retrospective study analyzed our experience at the Oral and Maxillofacial Surgery Department, University Hospital La Paz, Madrid (Spain), using the midline mandibulotomy approach in 67 patients, treated over a 15-year period between 2002 and 2017.

\section{Methods}

After obtaining the approval of the Ethics Committee of the University Hospital La Paz (Madrid, Spain), a chart review was performed for all patients who had undergone a mandibulotomy for access in the treatment of squamous cell carcinoma of the oral cavity and oropharynx between January 2002 and December 2017.

All charts were reviewed retrospectively for tumor type, stage and location, operative procedure performed (including the type of plate used), use of pre- or postoperative radiotherapy, length of follow-up, and evidence of complications.

Relevant Data were collected and deposit in a Data Base property of the Oral and Maxillofacial Surgery Department form the UNiversity Hospital La Paz in Madrid (Spain).

Data where analyzed with SPSS Statistics $\AA$ IBM $®$ v17.0. Differences in the rate of complications among the median and paramedian/lateral mandibulotomy group was determined by Fisher's exact test with a $p<0.005$ level o significance. .

\section{Results}

Sixty-seven patients underwent a mandibulotomy approach. There were 59 males (88\%) with a mean age of 56.9 years and eight females (12\%) with a mean age of 56.5 years. 
The primary tumor site was the tongue base (40.3\%), retromolar trigon and amygdala (22.3\%), floor of the mouth $(17.9 \%)$, oropharynx (10.4\%), and soft palate (7.5\%). [Table 1]

Table 1

Tumor site distribution

\begin{tabular}{|ll|}
\hline Tumor Site & No. of cases $^{\circ}$ \\
\hline Tongue & $27(40.3 \%)$ \\
\hline Retromolar trigon & $15(22.3 \%)$ \\
\hline Floor of the mouth & $12(17.9 \%)$ \\
Oropharynx & $7(10.4 \%)$ \\
Soft palate & $5(7.5 \%)$ \\
Total & 67 \\
\hline
\end{tabular}

The approach used was a midline mandibulotomy in 50 patients (74.6\%), a paramedian mandibulotomy in ten (14.9\%), and a lateral swing mandibulotomy in seven (10.44\%).[Table 2]

Table 2

Mandibulotomy distribution. The approach used was a midline mandibulotomy in 50 patients (74.6\%), a paramedian mandibulotomy in ten (14.9\%), and a lateral swing mandibulotomy in seven (10.44\%).

\begin{tabular}{|lll|}
\hline Type of Mandibulotomy & No. of Cases \\
\hline Median & & $50(74.6 \%)$ \\
\hline Paramedian & & $10(14.9 \%)$ \\
\hline Posterior & Body & $1(1.49 \%)$ \\
& Angle & $5(7.46 \%)$ \\
& Ramus & $1(1.49 \%)$ \\
& Total & $7(10.4 \%)$ \\
\hline Total & & 67 \\
\hline
\end{tabular}

In the patients undergoing median or paramedian mandibulotomies, adequate exposure of the lesion was achieved in all cases. With the lateral mandibulotomy approach, the senior surgeon noted limited exposure in 5 of 7 cases $(71 \%)$.

In 49 (73.13\%) patients, it was possible to perform an osteotomy without tooth extraction, while 18 $(26.86 \%)$ patients required an intraoperative tooth extraction to treat problems such as advanced dental 
caries and chronic periodontitis (10 patients [14.9\%]), to gain a safe surgical margin (4 patients [5.9\%]), or to obtain space for the osteotomy (5 patients [5.9\%]).

In all cases, rigid internal fixation with at least 2.0-mm titanium plates was performed. The most frequently used technique (56.6\%) was double plating with 2.0-mm plates (the upper plate with monocortical screws and the lower with bicortical screws) followed by double plating with a monocortical screw Unilock system (43\%).

Mandibulotomy-related complications were encountered in $16(23.8 \%)$ patients: 2 (2.9\%) were intraoperative, $4(6.0 \%)$ were within 3 weeks postoperatively, and $10(14.9 \%)$ were more than 3 weeks postoperatively. Of the 16 patients who developed complications, six (37.5\%), had mandibulotomies in the posterior mandible area, a higher rate statistically significant $(p<0.005)$ compared to the midline mandibulotomy group (13,7\%). [Table 3]

Table 3

Mandibulotomy complications

\begin{tabular}{|lll|}
\hline Timing & Complication & No. of cases \\
\hline \multirow{2}{*}{ Immediate } & Nerve Injury & $1(1.5 \%)$ \\
& Malocclusion & $1(1.5 \%)$ \\
& Orocutaneous fistula/Wound dehiscence & $2(2.9 \%)$ \\
\hline \multirow{2}{*}{ Late } & Infection & $2(2.9 \%)$ \\
\hline & Non-union & $4(6.0 \%)$ \\
\hline Total & Osteoradionecrosis & $4(6.0 \%)$ \\
\hline
\end{tabular}

\section{Discussion}

The location of the mandibulotomy in the midline offers some distinct advantages. First, it offers least disruption to the blood supply. Even though the mandibular symphysis normally has an overlapping blood supply from the inferior alveolar, lingual, and facial arteries, operative exposure often interrupts the latter two. A paramedian osteotomy forces reliance on the terminal branches of the inferior alveolar artery crossing the midline [7].

Second, since the osteotomy line is located far from the tumor, the total radiation dose to this location is lower than with lateral approaches, although a potential risk of osteoradionecrosis remains, even with the use of the new intensity modulated radiation therapy (IMRT) systems.

In our center, we try to minimize this risk using basic principles: we treat the patient's oral cavity prophylactically by extracting teeth that might be more likely to develop a postoperative infection; we use 
a thin saw blade to minimize the bone loss, enhancing the bone contact during osteosynthesis; we use generous irrigation during the osteotomy; and we perform internal rigid fixation with at least two 2.0-mm plates, separated by $2 \mathrm{~cm}$ in height, with bicortical screws in the lower plate and monocortical screws in the upper one. Furthermore, pre-adapting the plates to the symphyseal region before performing the osteotomy facilitates the posterior osteosynthesis and minimizes the incidence of malocclusions.

We recommend this kind of fixation for several reasons. Although some studies, like that of Amin et al. [7], recommend internal rigid fixation with just one 2.3-mm plate, clinical and experimental studies examining maxillofacial trauma have demonstrated the ability of two-plate rigid fixation at the symphyseal region to counteract the torsion forces generated at this point.

In our series, all the osteotomies were fixed with two 2.0-mm titanium plates with at least two holes on each side of the osteotomy. In $56.6 \%$ of the cases, the upper plate was fixed with monocortical screws and the lower one with bicortical screws. This allows rigid fixation with a low risk of damage to the teeth apex. However, over the last 4 years we have made extensive use of plates with a lock system (43\%) (Figure 4). This also provides a rigid fixation, acting as an external-internal fixation device, and it does not require perfect adaptation to the bone.

Good bony apposition is necessary for direct bone healing. Since most of the patients will receive postoperative radiotherapy, it is important to accelerate the healing process as much as possible.

Another risk factor for the development of complications is the use of preoperative radiotherapy. Komisar et al. [8], reported that a significant percentage $(71.4 \%)$ of the patients who underwent a mandibular osteotomy following radiotherapy developed postoperative complications, seen as osteoradionecrosis or malunion. In such cases, this procedure should be avoided whenever possible. If indispensable, then meticulous preoperative and postoperative management of the oral status is required.

Dubner and Spiro [9], reported a 20\% complication rate and Shah et al. [10] reported complications such as bone sepsis, wound sepsis, fistulas, delayed union, and cases that required the removal of the wire or fixation plate. By contrast, McCann et al. [11] reported that $35 \%$ of the patients in whom rigid fixation was used after the mandibulotomy developed an immediate postoperative infection, osteoradionecrosis, plate exposure, plate fracture, or non-union.

In our series, the rate of complications (23.8\%) was like reported rates, [12], with a greater incidence using the posterior mandibulotomy approach $(20.8 \%)$.

In our experience, with a careful incision design, good mandibular osteosynthesis and proper muscle reattachment aesthetic sequalae are usually negligible in most patients (Figure 5).

There is no doubt that the future of oral surgery leads toward minimally invasive approaches such as the use of Robotic Surgery for base of the tongue and oro/hypopharynx tumors, [13] with less morbidity [14]. However, until this technology is not widespread, conventional techniques such as midline 
mandibulotomy approaches, with "modern-times" refinements, such as those described previously in the article, still have their place in head and neck oncology surgery.

\section{Conclusions}

In our experience, a median straight mandibulotomy is a safe, easy procedure with acceptable morbidity. It provides excellent exposure for oral and oropharyngeal tumors, with a low complication rate, significantly lower, as shown in our study, compared to the more posterior paramedian or lateral approaches.. A thin saw blade should be used to minimize the bone loss and improve the bone contact. Rigid fixation with two 2.0-mm titanium plates with a lock system is highly recommended as it improves bone fragments apposition.

Until minimally invasive Robotic technology is not widespread, conventional techniques such as midline mandibulotomy approaches, with "modern-times" refinements still have their place in head and neck oncology surgery

\section{Abbreviations}

IMRT: Intensity Modulated Radiation Therapy.

\section{Declarations}

Ethics approval and consent to participate: The retrospective chart review study was approved by the Ethics Committee of the University Hospital la Paz in 2017.

Consent for publication: Consent for publication was granted by all the patients included in the study.

Availability of data and materials: The datasets used and/or analyzed during the current study belong to the Oral and Maxillofacial Surgery Department, University Hospital la Paz (Madrid, Spain). They are available from the corresponding author on reasonable request.

Competing interests. This research received no external funding. The authors declare that they have no competing interests

Funding: No funding was granted for this study. The authors declare no conflict of interest.

Authors' contributions: Conceptualization and methodology, JMLA.; investigation, JLDC; resources, JLC.; data curation, writing-original draft preparation, JMV, BRS-review and editing, PAL.; supervision, JMA.

Acknowledgements. Not applicable

Authors information: 
José M López-Arcas MD, DDS, MS PhD; Juan Manuel Vadillo, DDS, $\mathrm{PhD}^{2}$; José L Del Castillo MD, PhD ${ }^{3}$; Patricia A Lara DDS, PhD 4; José L. Cebrián, MD, DDS, MS, $\mathrm{PhD}^{5}$, Beatriz Ruiz-Sánchez, $\mathrm{DDS}^{6}$, Juan M Aragoneses, MD, DDS, MS, $\mathrm{PhD}^{7}$.

1. Consultant .Maxillofacial Surgery Department. University Hospital La Paz (Madrid, Spain), Radiology Department. University Hospital Infanta Leonor (Madrid, Spain), Dentistry Faculty. Alfonso X el Sabio University (Madrid, Spain): jmloparc78@gmail.com

2. Director. Master in Oral Surgery and Periodontics. Alfonso X el Sabio University (Madrid, Spain). jumava10@gmail.com

3. Head of the TMJ Unit. Maxillofacial Surgery Department. University Hospital La Paz (Madrid, Spain) delcastillo6@hotmail.com

\section{Academic Coordinator. Dentistry School. Alfonso X el Sabio University (Madrid, Spain). plaralpe@uax.es}

5. Chief of Service. University Hospital La Paz (Madrid, Spain): rodrigator2001@hotmail.com

6. University Clinic Coordinator. Alfonso X el Sabio University (Madrid, Spain). Bearuiz146@gmail.com

7. Dean. Dentistry School. Alfonso X el Sabio University (Madrid, Spain). jaraglam@uax.es

All authors have read and agreed to the published version of the manuscript.

All methods were performed in accordance with the relevant guidelines and regulations.

Informed consent from all subjects were obtained for both study participation and publication of identifying information/images

\section{References}

1. Butlin HT. Diseases of the Tongue. Clinical Manuals for Practitioners and Students of Medicine. London: Cassell; 1885; pp

2. Spiro RH, Gerold FP, Strong EW. Mandibular "swing" approach for oral and oropharyngeal tumors. Head Neck Surg 198; Vol;3, pp. 371-378.

3. Spiro RH, Gerold FP, Shah JP, et al. Mandibulotomy approach to oropharyngeal tumors. Am J Surg 1985, Vol 150, pp.466-469.

4. Nam W, Kim HJ, Choi EC, Kim MK, Lee EW, Cha IH. Contributing factors to mandibulotomy complications: A retrospective study. Oral Surg Oral Med Oral Pathol Oral Radiol Endod 2006;Vol 101, pp. E65-70.

5. Singh AM, Bahadur S, Tandon DA, Pande RM. Anterior mandibulotomy of oral and oropharyngeal tumours. J Laryngol Otol 1993; Vol 107, pp.316-319. 
6. Sardi A, Walters PJ. Modified mandibular swing procedure for resection of carcinoma of the oropharynx. Head Neck 1991 Vol 13, pp.394-397.

7. Amin MR, Deschler DG, Hayden RE. Straight midline mandibulotomy revisited. Laryngoscope 1999; Vol 109, pp.1402-1405.

8. Komisar A, Shapiro BM. Complications of midline mandibulotomy. Ear Nose Throat J 1988;Vol 67, pp. 521-523.

9. Dubner S, Spiro RH. Median mandibulotomy: a critical assessment. Head Neck 1991; Vol 13, pp.389-393.

10. Shah JP, Kumaraswamy SV, Kulkarni V. Comparative evaluation of fixation methods after mandibulotomy for oropharyngeal tumors. Am J Surg 1993;Vol 166, pp.431-434.

11. McCann KJ, Irish JC, Gullane PJ. Complications associated with rigid fixation of mandibulotomies. J Otolaryngol 1994; Vol 23, pp.210-215.

12. Dai TS, Hao SP, Chang KP, Pan WL, Yeh HC, Tsang NM. Complications of mandibulotomy: Midline versus paramidline. Otolaryngol Head Neck Surg 2003;Vol 128, pp. 137-141.

13. Holsinger FC, Ferris RL. Transoral endoscopic head and neck surgery and its role within the multidisciplinary treatment paradigm of oropharynx cancer: robotics, lasers and clinical trials. J Clin Oncol 2015; Vol 33, pp. 3285-3292

14. Jolly SS, Rattan V, Verma U. Traumatic posterior dislocation of bilateral mandible condyles into external auditory canal treated with midline mandibulotomy: a rare case report and review of the literature. DOI: 10.1007/s12663-020-01442-8

\section{Figures}




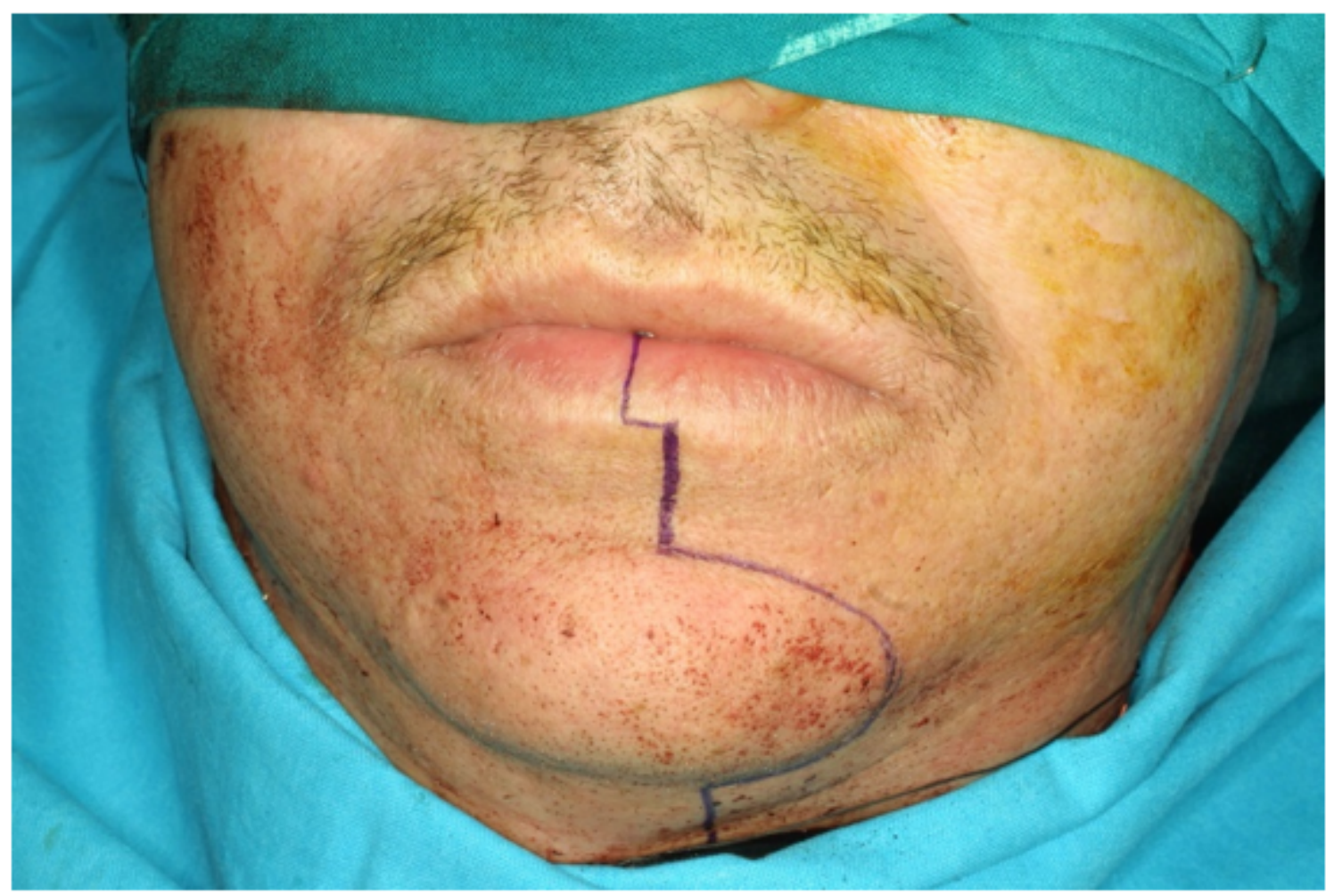

Figure 1

Skin incision design.

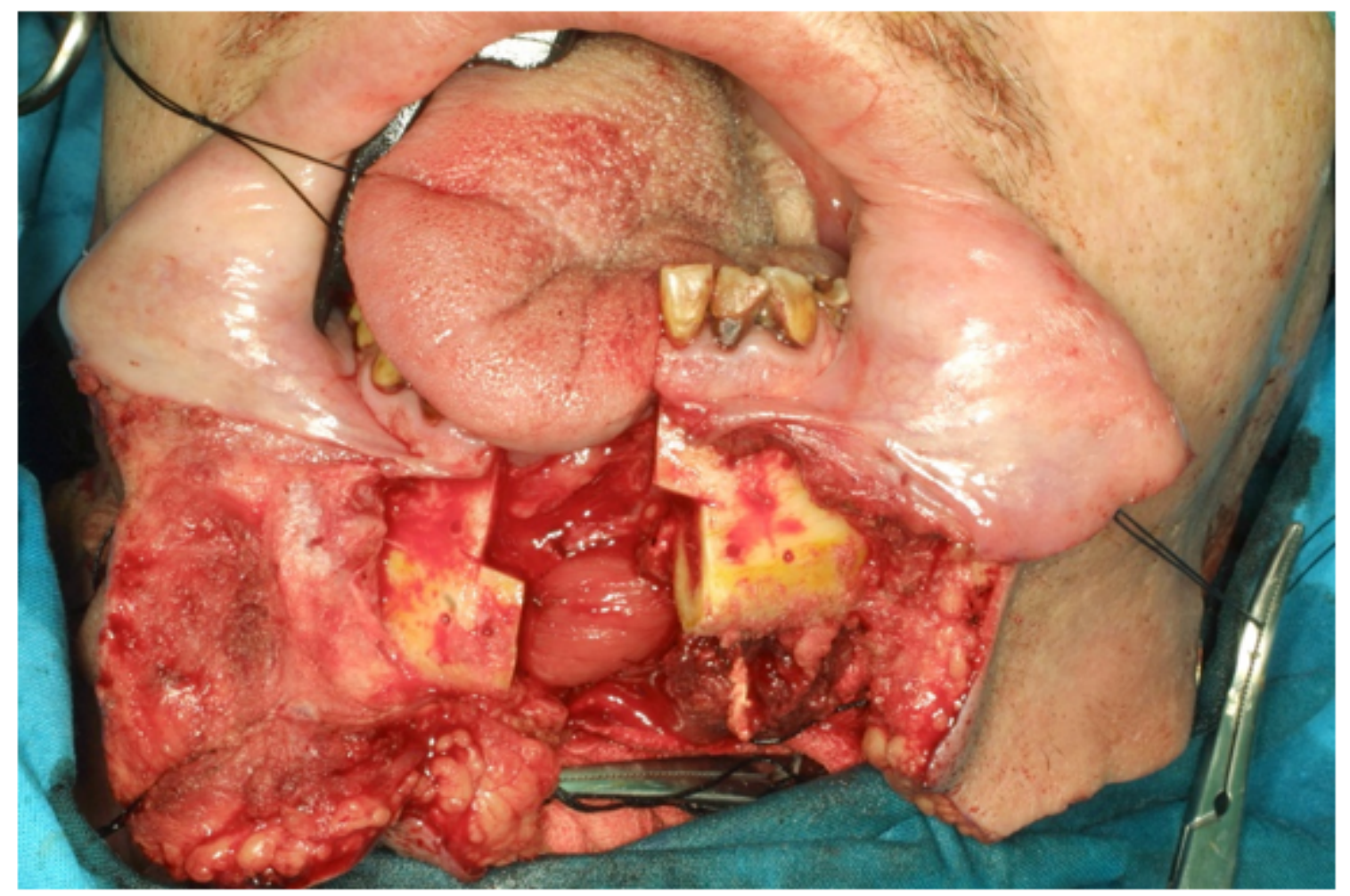

Figure 2 
Stair-step midline mandibulotomy.

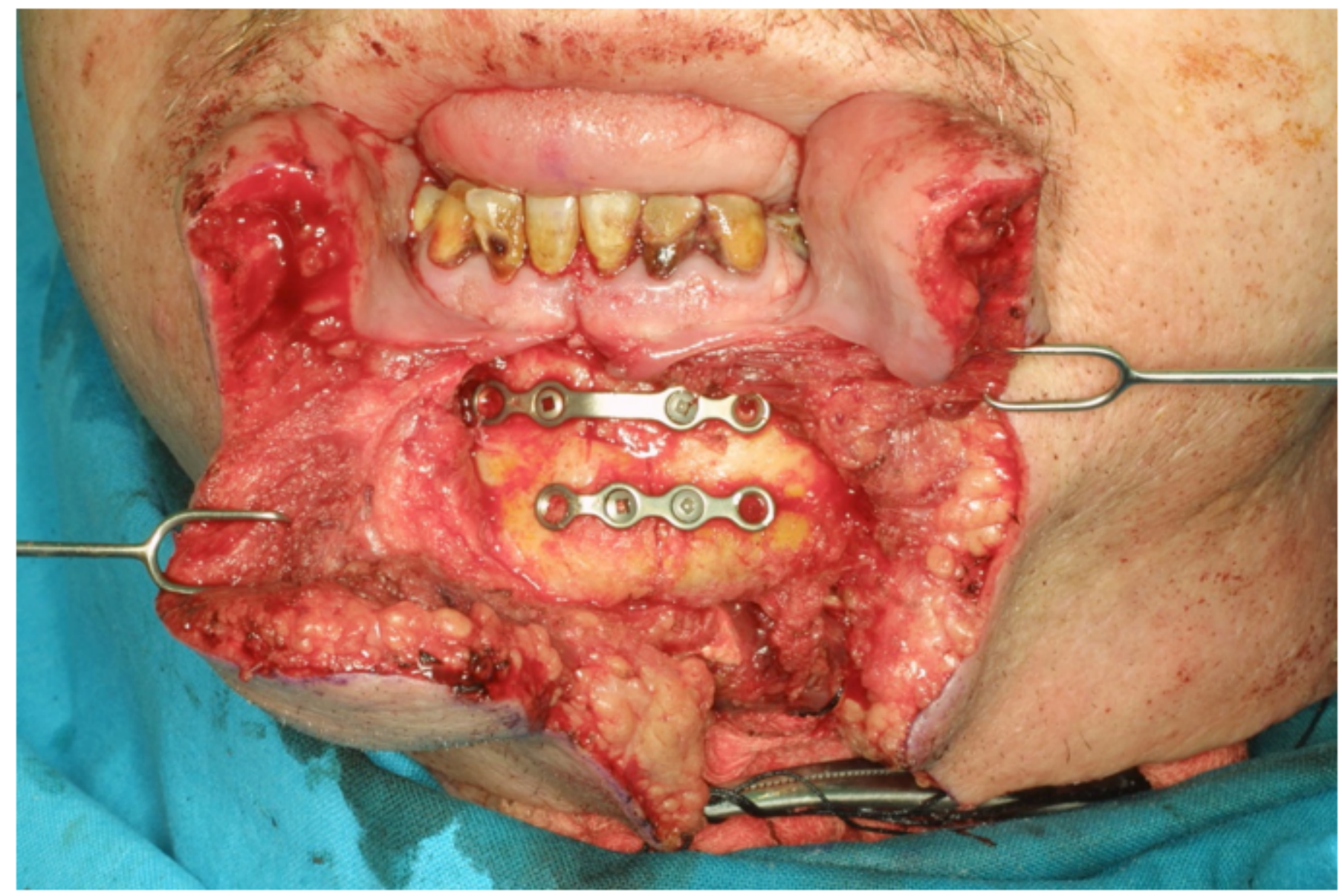

Figure 3

Midline osteotomy osteosynthesis with two miniplates $2.0 \mathrm{~mm}$. 


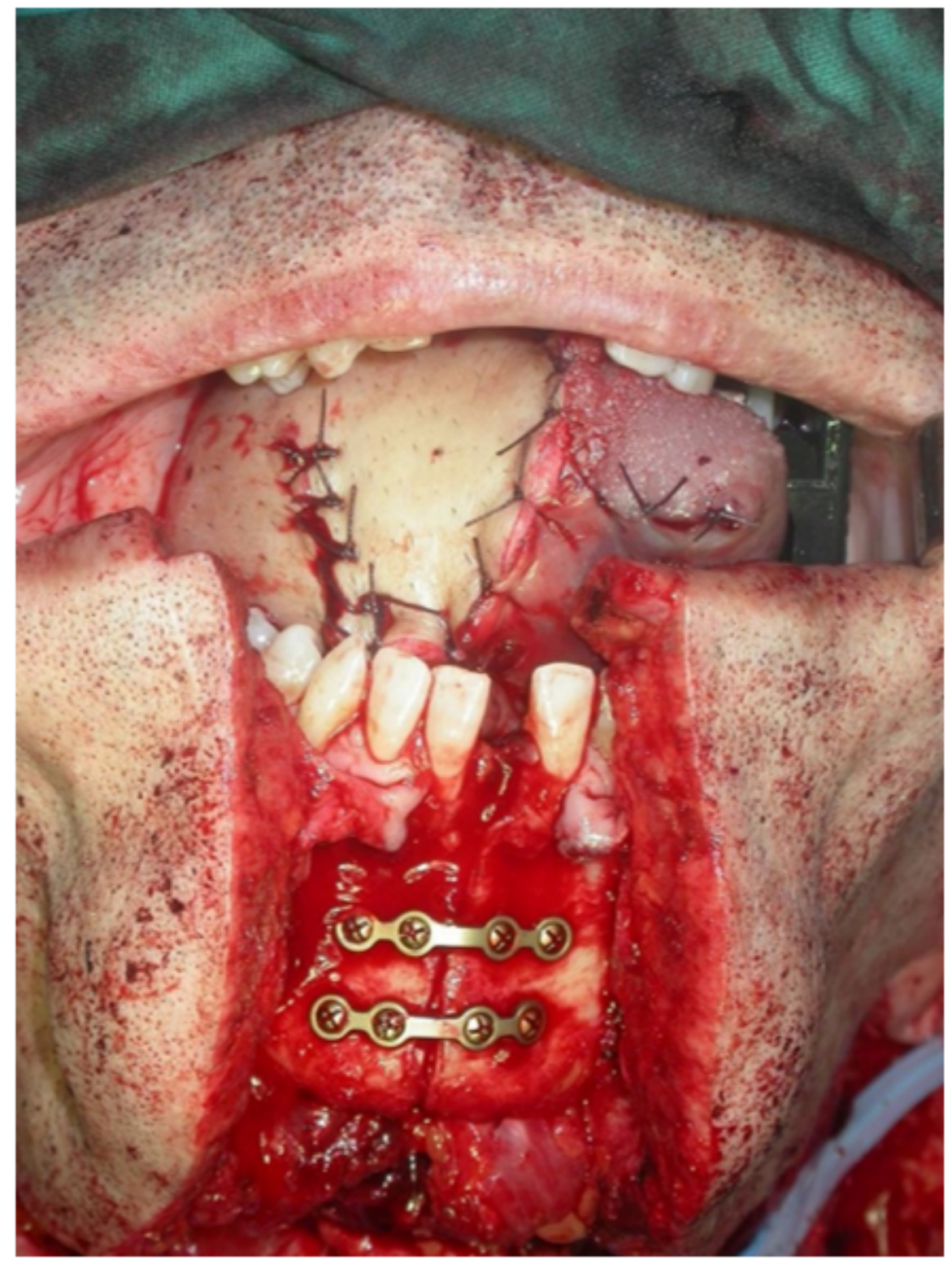

\section{Figure 4}

Midline mandibulotomy. Osteosynthesis with two plates "lock" system $2.0 \mathrm{~mm}$. 


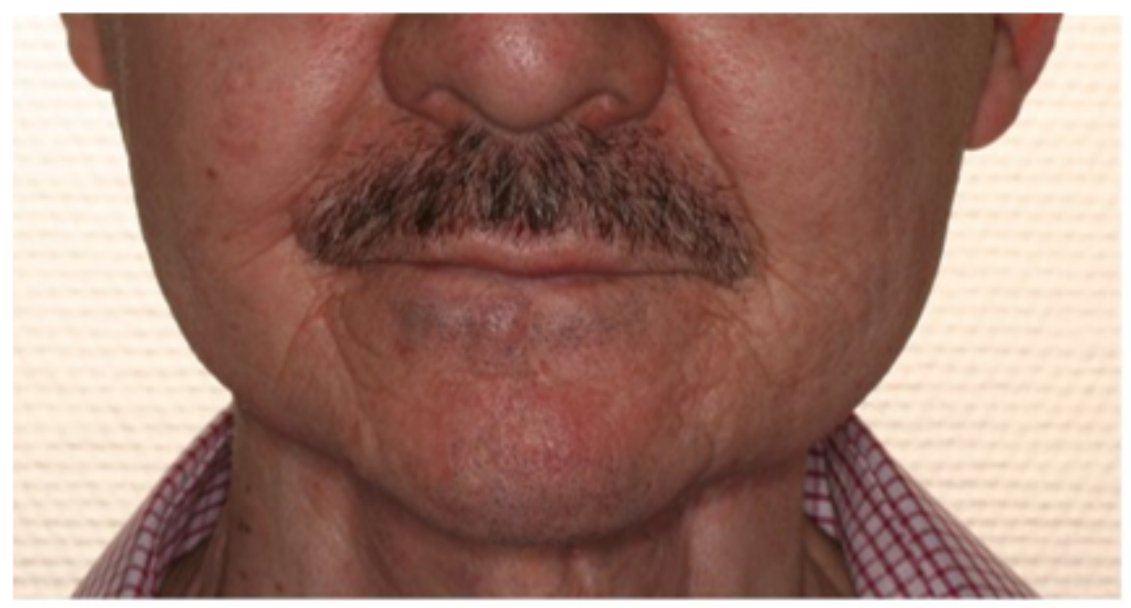

Figure 5

Long term appearance after the mandibulotomy. 